\title{
Psychological Burden amongst Caregivers of Patients on Maintenance Haemodialysis
}

\author{
Abdul Rehman Arshad ${ }^{1}$, Taleah Tahir ${ }^{2}$, Abdul Wahab Mir ${ }^{2}$ and Salahuddin ${ }^{2}$ \\ ${ }^{1}$ Department of Nephrology, Combined Military Hospital, Peshawar, Pakistan \\ ${ }^{2}$ Department of Nephrology, Pak Emirates Military Hospital, Rawalpindi, Pakistan
}

\begin{abstract}
Long-term caregiving is a difficult job, risking mental health of involved individuals. This study was planned to document magnitude of the problem at Pak Emirates Military Hospital, Rawalpindi, with an aim to improve quality of life in caregivers and patients alike. From January to June 2019, caregivers accompanying haemodialysis patients were selected using consecutive sampling technique. Those unwilling were excluded. Psychological burden was assessed using Zarit Burden Interview (ZBI) questionnaire. There were 98 caregivers, including $71(72.45 \%)$ males and $27(27.55 \%)$ females, aged $33.13 \pm 9.98$ years. There was little/ no burden in 27 (27.55\%) caregivers; $52(53.06 \%)$ had mild to moderate, 18 (18.37\%) had moderate to severe and 3 (3.06\%) had severe psychological burden. Mean ZBI score was $30.00 \pm 12.57$. Males had lower scores than females (28.01 \pm 11.18 vs. $35.22 \pm 14.65$, respectively; $p=0.026$ ). Gender of caregivers significantly predicted ZBI scores; while their age, job status, education, monthly income and duration of caregiving did not.
\end{abstract}

Key Words: Caregivers, Chronic kidney disease, Haemodialysis, Long-term care, Psychological burden.

How to cite this article: Arshad AR, Tahir T, Mir AW, S. Psychological Burden amongst Caregivers of Patients on Maintenance Haemodialysis. J Coll Physicians Surg Pak 2021; 31(06):743-745.

The burden of end-stage renal disease (ESRD) is increasing progressively, proportionate to the growing prevalence of major risk factors including diabetes and hypertension. ${ }^{1}$ Renal transplant is the treatment of choice, known to improve both morbidity and mortality. Many patients cannot afford this because of financial constraints or shortage of donors. This leaves them with the only option of long-term haemodialysis (HD). They have to attend in-centre HD sessions three times a week for an indefinite period, a difficult task. Caregivers play an important role in looking after patients with ESRD. This could include taking care of their needs at home as well as accompanying them to HD centre. These roles assume greater importance in Pakistan because of cultural values. While managing patients, we often ignore their family members. Another important aspect is the perception of caregivers' contributions by the patients themselves. This is significant as this could affect the quality of life of the patients themselves. Whereas, we now have a lot of local data assessing psychiatric morbidity amongst patients with ESRD, not many studies from Pakistan have looked into the problems faced by theircaregivers.

Correspondence to: Dr. Abdul Rehman Arshad, Department of Nephrology, Combined Military Hospital, Peshawar, Pakistan

E-mail: maj.abdulrehman@gmail.com

Received: July 20, 2020; Revised: December 08, 2020;

Accepted: January 08, 2021

DOI: https://doi.org/10.29271/jcpsp.2021.06.743
We, therefore, planned this study to determine the magnitude of the problem in our setup. The results would help create more awareness and open avenues for treatment of stressed caregivers. Such an action would indirectly improve outcomes for ESRD patients as well.

This cross-sectional study was carried out at the Department of Nephrology, Pak Emirates Military Hospital, Rawalpindi, from January to June 2019 after obtaining approval from the local Ethics Review Committee (Reference number A/28/ PEMH/EC-19/21). We enlisted all patients who had been on maintenance HD at our centre for at least three months and were accompanied by caregivers at the HD unit. We invited their caregivers (only family members living with the patients) for inclusion in this study, using consecutive sampling technique.

Only one caregiver for any given patient on HD was approached. Caregivers not providing consent, those known to have anxiety, depression or any other psychiatric illness, domestic servants and those helping the patients only with transportation to and from HD unit, were excluded. A minimum sample size of 97 was calculated with Free Statistics Calculators version 4.0 , using an anticipated effect size $\left(\mathrm{f}^{2}\right)$ of 0.15 , power of 0.8 , alpha of 0.05 and 6 predictors. We collected demographic data on six factors that could potentially predict psychological burden in the caregivers: their age, gender, occupation, level of education, monthly income and the time period they had been coming to the hospital as caregivers. 


Table I: Mean scores for individual items of Zarit Burden Interview.
\begin{tabular}{|l|l|l|}
\hline Item No & Question & Score \\
\hline 1 & Do you feel that your relative asks for more help than he/she needs? & $1.36 \pm 0.89$ \\
\hline 2 & Do you feel that because of the time you spend with your relative that you don't have enough time for yourself? & $1.27 \pm 0.95$ \\
\hline 3 & Do you feel stressed between caring for your relative and trying to meet other responsibilities for your family or work? & $1.31 \pm 0.95$ \\
\hline 4 & Do you feel embarrassed over your relative's behaviour? & $1.26 \pm 0.99$ \\
\hline 5 & Do you feel angry when you are around your relative? & $1.42 \pm 0.99$ \\
\hline 6 & Do you feel that your relative currently affects our relationships with other family members or friends in a negative way? & $1.41 \pm 0.93$ \\
\hline 7 & Are you afraid what the future holds for your relative? & $1.58 \pm 1.03$ \\
\hline 8 & Do you feel your relative is dependent on you? & $1.42 \pm 0.99$ \\
\hline 9 & Do you feel strained when you are around your relative? & $1.40 \pm 1.00$ \\
\hline 10 & Do you feel your health has suffered because of your involvement with your relative? & $1.43 \pm 1.05$ \\
\hline 11 & Do you feel that you don't have as much privacy as you would like because of your relative? & $1.49 \pm 1.04$ \\
\hline 12 & Do you feel that your social life has suffered because you are caring for your relative? & \\
\hline 13 & Do you feel uncomfortable about having friends over because of your relative? & \\
\hline 14 & Do you feel that your relative seems to expect you to take care of him/her as if you were the only one he/she could depend on? & $1.37 \pm 1.07$ \\
\hline 15 & Do you feel that you don't have enough money to take care of your relative in addition to the rest of your expenses? & $1.50 \pm 1.09$ \\
\hline 16 & Do you feel that you will be unable to take care of your relative much longer? & \\
\hline 17 & Do you feel you have lost control of your life since your relative's illness? & $1.29 \pm 0.95$ \\
\hline 18 & Do you wish you could leave the care of your relative to someone else? & $1.20 \pm 0.94$ \\
\hline 19 & Do you feel uncertain about what to do about your relative? & $1.20 \pm 0.96$ \\
\hline 20 & Do you feel you should be doing more for your relative? & $1.35 \pm 1.10$ \\
\hline 21 & Do you feel you could do a better job in caring for your relative? & $1.26 \pm 1.02$ \\
\hline 22 & Overall, how burdened do you feel in caring for your relative? & $1.12 \pm 1.05$ \\
\hline
\end{tabular}

Table II: Results of standard multiple regression.

\begin{tabular}{|l|l|l|l|}
\hline Variable & Unstandardised coefficient (B, 95\% Cl) & Standardised coefficient (Beta) & p-value \\
\hline Age & $0.224(-0.059,0.506)$ & 0.178 & 0.119 \\
\hline Gender & $8.638(1.534,15.742)$ & 0.308 & 0.018 \\
\hline Job status & $5.044(-2.166,12.254)$ & 0.190 & 0.168 \\
\hline Education & $0.305(-0.409,1.019)$ & 0.091 & 0.398 \\
\hline Income & $<0.001(<0.001,<0.001)$ & -0.119 & 0.400 \\
\hline Duration of caregiving & $-0.035(-0.142,0.072)$ & -0.066 & 0.517 \\
\hline
\end{tabular}

Psychological burden was assessed using ZBI. This questionnaire contains 22 items, each having five responses scored from 0 to 4 . Total scores up to 21 indicate little or no burden, 21-40 indicate mild to moderate burden, 41-60 indicate moderate to severe burden, whereas $61-88$ reflect severe burden.

The questionnaire was translated to Urdu and then back-translated to English by two separate doctors. The original and back-translated version were compared by the first author to ensure that no significant difference had emerged during this process. We then used the Urdu version for this study. Patients literate enough to comprehend the questionnaire self-reported. Others were asked all the questions in direct face-to-face interviews by the second author.

Data was analysed using IBM SPSS Statistics for Windows, Version 20.0 (IBM Corp, Armonk, NY). Quantitative data with parametric distribution was described as mean \pm standard deviation (SD), whereas non-parametric data was described as median and range. Standard multiple regression was carried out to determine the relationship between ZBI scores and the six possible predictors. $P$ values $\leq 0.05$ were considered statistically significant.

The mean age of 98 caregivers was $33.13 \pm 9.98$ years. There were $71(72.45 \%)$ males and $27(27.55 \%)$ females. Most $(n=66 ; 67.35 \%)$ were working, while $32(32.65 \%)$ did not have any job. Mean education level was $10.88 \pm 3.72$ years. Median monthly income was Rs. 18,000 (range: 0180,000 ). Mean attendance vintage was 12 months (range: $0-120$ months). Majority of the attendants ( $n=90 ; 91.84 \%)$ accompanied patients on more than $50 \%$ of the sessions, whereas the rest $(8 ; 8.16 \%)$ were present during less than $50 \%$ sessions. There was little/no psychological burden in 27 (27.55\%) caregivers; 52 (53.06\%) had mild to moderate, 18 (18.37\%) had moderate to severe and $3(3.06 \%)$ had severe psychological burden. Mean ZBI score was $30.00 \pm 12.57$. Mean scores for individual items of this scale are shown in Table I. Males had lower scores than females $(28.01 \pm 11.18$ vs. $35.22 \pm 14.65$, respectively; $p=0.026$ ). As shown in Table II, gender of the caregivers was the only factor significantly predicting total ZBI sores.

Caregivers are almost universally required for ESRD patients considering their advanced age, presence of multiple co-morbidities and intricacies of HD. Looking after these patients is an overwhelming experience, with greater expectations keeping in view the traditional Eastern cultural values. Close family members of HD patients are thus an integral part of the management team, sharing important responsibilities in care. This study has highlighted psychological burden in a vast majority $(72.45 \%)$ of caregivers. Most of them perceived only mild to moderate burden. Our results are comparable to figures reported in a study done at other dialysis setups in Rawalpindi five years ago. ${ }^{2}$ Caregiving burden was noted in $80 \%$ of those cases, mostly mild to moderate. 
Left unaddressed, this burden would eventually translate into poor quality of life.

Majority of the caregivers were males. The male preponderance in our study population could be because of the physical strength required to move patients between home and dialysis centre. It is very much possible that the roles get changed at homes, where females could look after other needs of these patients. We do not have further information on this for our study population. Female caregivers had higher ZBI scores in this study, similar to results of a Turkish study. ${ }^{3}$ The most plausible explanation would be the stress of looking after patients in addition to the responsibility of carrying out household chores and taking care of other family members. Strangely, none of the other parameters studied was associated with psychological burden. The results are in contrast to generally available literature. Farzi et al. documented greater burden in working caregivers from Iran. ${ }^{4}$ It is very much possible that the caregivers in our cohort had adopted coping strategies to minimise the impact of work on their daily lives. Caregivers' income also did not have any relationship with psychological burden in this study. Similar results were reported by Hoang et al. in a study done from Vietnam; ${ }^{5}$ whereas, Mashayekhi et al. described a significant relationship of income with caregiver burden score amongst Irani patients. ${ }^{6}$

ZBI has been used to assess caregiver burden in a number of clinical conditions. Amongst caregivers of patients with heart failure, it has been shown to have a good internal consistency (Cronbach's $\alpha=0.921$ ) with good item-total ( $r=$ $0.395-0.764$ ) and item-item (mean $r=0.365$ ) correlations. $^{7}$

Apart from the inherent problems associated with a cross-sectional study design, this study is limited by the absence of a control group comprising of healthy individuals. It is generally believed that the total time spent caring for patients in a day is a predictor of psychological burden. ${ }^{5}$ We focused only on caregivers' attendance at HD sessions and did not record data about relevant activities while at home. Moreover, we do not have data to suggest the extent to which the patients could care for themselves. Data on transportation used by patients is also missing. Some of the caregivers had to be interviewed as they could not self-administer the questionnaire. This could have produced information bias.

In short, caregivers' psychological burden is a significant problem in haemodialysis patient populations, more so amongst females. This necessitates periodic screening, with an aim to help affected persons in reducing the mental stress.

\section{ETHICAL APPROVAL:}

Ethical approval was obtained from Ethical Committee of Pak
Emirates Military Hospital, Rawalpindi, vide approval letter No. A/28/PEMH/EC-19/21.

\section{PATIENTS' CONSENT:}

Informed written consents were obtained from all study participants.

\section{CONFLICT OF INTEREST:}

The authors declared no conflict of interest.

\section{AUTHORS' CONTRIBUTION:}

ARA: Interpreted data, critically revised the manuscript, approved the final version, agreed to be accountable for all aspects.

TT: Conceived the study, analysed data, drafted the manuscript, approved the final version, agreed to be accountable for all aspects.

AWM: Designed the study, acquired data, drafted the manuscript, approved the final version, agreed to be accountable for all aspects.

SU: Analysed data, drafted the manuscript, approved the final version, agreed to be accountable for all aspects.

\section{REFERENCES}

1. GBD chronic kidney disease collaboration. Global, regional, and national burden of chronic kidney disease, 1990-2017: A systematic analysis for the global burden of disease study 2017. Lancet 2020; 395(10225):709-33. doi: 10.1016/ S0140-6736(20)30045-3.

2. Usman Shah HB, Atif I, Rashid F, Babar MW, Arshad F, Qamar W, et al. Assessment of caregiver burden of patients receiving dialysis treatment in Rawalpindi. J Pak Med Assoc 2017; 67(10):1498-501.

3. Cagan O, Unsal A, Celik N, Yilmaz AT, Culha I, Eren HK. Care burden of caregivers of hemodialysis patients and related factors. Int J Caring Sci 2018; 11: 279-84.

4. Farzi S, Farzi S, Moladoost A, Ehsani M, Shahriari M, Moieni $M$. Caring burden and quality of life of family caregivers in patients undergoing hemodialysis: A descriptive-analytic study. IJCBNM 2019; 7(2):88-96. doi: 10.30476/ IJCBNM.2019.44888.

5. Hoang VL, Green T, Bonner A. Informal caregivers of people undergoing haemodialysis: Associations between activities and burden. J Ren Care 2019; 45(3):151-8. doi: 10.1111/ jorc. 12280.

6. Mashayekhi F, Pilevarzadeh M, Rafati F. The assessment of caregiver burden in caregivers of hemodialysis patients. Mater Sociomed 2015; 27(5):333-6. doi: 10.5455/msm. 2015. 27.333-336.

7. Al-Rawashdeh SY, Lennie TA, Chung ML. Psychometrics of the Zarit Burden Interview in caregivers of patients with heart failure. J Cardiovasc Nurs 2016; 31(6):e21-e8. doi: $10.1097 / J C N .0000000000000348$. 